\title{
OBSIDIANA VERDE EN LA ESTEPA FUEGUINA: UN HALLAZGO EN AMALIA 4
}

\author{
JIMENA ORÍA*, MÓNICA C. SALEMME** Y FERNANDO C. SANTIAGO*
}

\begin{abstract}
RESUMEN
En este trabajo se describe el contexto del sitio de superficie Amalia 4, referenciando un hallazgo particular: una punta de obsidiana verde que ofrece la oportunidad de sugerir, a partir del análisis morfotecnológico, un marco cronológico, que en este tipo de contextos resulta un desafío para el estudio de la arqueología regional. El registro de obsidiana verde en este sitio de la estepa fueguina, aporta nuevos datos a la discusión sobre la distribución espacial y temporal de esta materia prima.
\end{abstract}

PALABRAS CLAVES: obsidiana verde, estepa fueguina, sitio de superficie, intercambio. GREEN OBSIDIAN IN THE FUEGIAN STEPPE: THE FINDING IN AMALIA 4

\section{ABSTRACT}

This paper describes the context of the surface site Amalia 4, regarding a particular data: a green obsidian projectile point that offers the opportunity to suggest a chronological framework, based on the technomorphological analysis. The chronological location of these contexts becomes a challenge for the study of regional archaeology. The finding of green obsidian in a site of the Fuegian steppe provides new data for the discussion on the spatial and chronological distribution of this raw material.

KEYWORDS: green obsidian, fuegian steppe, surface site, exchange.

Lab. de Geomorfología y Cuaternario, CADIC-CONICET. B. Houssay 200. Ushuaia, Argentina; jimenaoria@hotmail.com; ersant2@gmail.com

* Lab. de Geomorfología y Cuaternario, CADIC-CONICET. B. Houssay 200. Ushuaia; Universidad Nacional de la Patagonia San Juan Bosco, Sede Ushuaia, Argentina; msalemme@cadic-conicet.gob.ar 


\section{INTRODUCCIÓN}

En el sector interior de la estepa del norte de la Isla Grande de Tierra del Fuego, donde la vegetación típica de coironal genera una situación de visibilidad nula en casi toda la superficie del terreno, es un rasgo común la formación de hoyadas de deflación en distintos sectores del paisaje, como producto de la acción eólica y la incidencia del ganado ovino (Santiago y Oría 2007, Oría et al. 2010). En estas hoyadas es frecuente el hallazgo de material arqueológico en superficie, a veces con altas

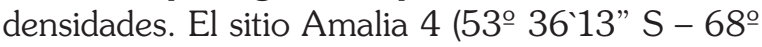
$28^{\prime} 05^{\prime \prime}$ O, Fig. 1a) es un ejemplo de este tipo de contextos; en la costa sur de la laguna homónima se abre una cubeta de deflación de aproximadamente $600 \mathrm{~m}^{2}$ de superficie, dejando expuesto material lítico y restos óseos.

$\mathrm{Al}$ analizar los frecuentes contextos de superficie en la estepa fueguina, nos enfrentamos al problema de establecer un marco cronológico para los materiales allí registrados. Fanning y Holdaway (2001) y Holdaway et al. (2004) ubican cronológicamente este tipo de sitios cruzando información proveniente de dos vías: a) la edad máxima de los sedimentos sobre los que se encuentran los materiales y b) considerando características propias del conjunto en relación a otros conjuntos recuperados en estratigrafía (con fechados conocidos), información etnográfica o bien análisis tafonómicos de los materiales. En nuestro caso, hasta el momento no contábamos con ningún material que pudiera funcio- nar como indicador cronológico y ofrecer un marco, al menos relativo, a los conjuntos recuperados en superficie en el área de estudio.

\section{AMALIA 4, CONTEXTO DE HALLAZGO}

Como se mencionó, el sitio se ubica en la margen meridional de la laguna homónima, en una gran hoyada de deflación. Identificada esta cubeta, se relevó su topografía y se recolectó la totalidad de materiales en superficie (Fig. 1b). El conjunto recuperado está compuesto por artefactos líticos $(\mathrm{N}=129)$ y restos faunísticos $(\mathrm{N}=35)$, presentando estos últimos, en general, estadios de meteorización avanzados.

Los desechos lato sensu componen el 83,7\% $(\mathrm{N}=108)$ de la muestra lítica, de los cuales los más representados son las lascas $(\mathrm{N}=54)$, seguidas de los desechos indiferenciados $(\mathrm{N}=30)$, núcleos $(\mathrm{N}=23)$ y nódulos ( $\mathrm{N}=1$ ) (Tabla 1$)$. El 16,3\% restante lo componen instrumentos: raederas, raspadores, una preforma y una punta. La tabla 1 consigna, en frecuencias absolutas y porcentajes, la composición artefactual del conjunto discriminando por materia prima; resulta llamativa la elevada proporción de artefactos formatizados de la muestra, rasgo poco común en los conjuntos de la región. Entre los instrumentos son las raederas las que predominan $(\mathrm{N}=16)$, seguida de raspadores $(\mathrm{N}=3)$, preforma y punta ( $\mathrm{N}=1$ ambas categorías).

Por el momento no fue posible constatar remontajes líticos, pero sí la existencia de artefac-

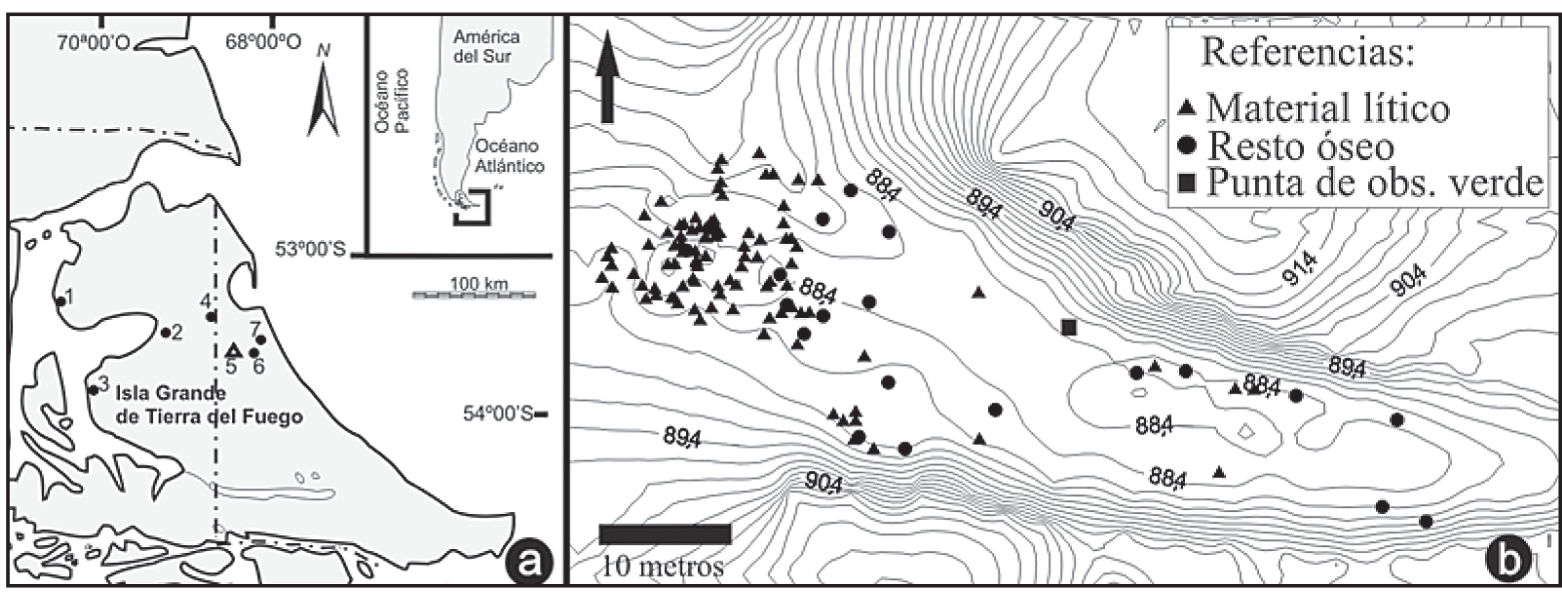

Fig. 1. a) Ubicación aproximada de sitios mencionados. 1: Porvenir Norte 28; 2: localidad Marazzi; 3: Río Caleta 4; 4: Puesto Consuelo 3; 5: Amalia 4; 6: Laguna Grande; 7: Avilés 3; b) topografía y distribución de materiales del sitio Amalia 4. 
Tabla 1. Amalia 4, composición artefactual del conjunto lítico.

\begin{tabular}{|c|c|c|c|c|c|c|c|c|c|c|c|c|c|c|c|}
\hline M. Prima / Especimen & \multicolumn{2}{|c|}{ Calcedonia } & \multicolumn{2}{|c|}{ Riolita } & \multicolumn{2}{c|}{ Basalto } & \multicolumn{2}{c|}{ Cuarzo } & \multicolumn{2}{c|}{ Obsidiana } & \multicolumn{2}{|c|}{ Indet } & \multicolumn{2}{|c|}{ Total } \\
\hline & $\mathrm{N}$ & $\%$ & $\mathrm{~N}$ & $\%$ & $\mathrm{~N}$ & $\%$ & $\mathrm{~N}$ & $\%$ & $\mathrm{~N}$ & $\%$ & $\mathrm{~N}$ & $\%$ & $\mathrm{~N}$ & $\%$ \\
\hline Nódulo & 1 & 0,8 & 0 & 0 & 0 & 0 & 0 & 0 & 0 & 0 & 0 & 0 & 1 & 0,8 \\
\hline Núcleo & 4 & 3,1 & 15 & 11,6 & 1 & 0,8 & 0 & 0 & 0 & 0 & 3 & 2,3 & 23 & 17,8 \\
\hline Lasca & 13 & 10,1 & 27 & 20,9 & 10 & 7,8 & 0 & 0 & 0 & 0 & 4 & 3,1 & 54 & 41,9 \\
\hline Desecho indiferenciado & 16 & 12,4 & 8 & 6,2 & 3 & 2,3 & 1 & 0,8 & 0 & 0 & 2 & 1,6 & 30 & 23,3 \\
\hline Preforma & 1 & 0,8 & 0 & 0 & 0 & 0 & 0 & 0 & 0 & 0 & 0 & 0 & 1 & 0,8 \\
\hline Raedera & 5 & 3,9 & 7 & 5,4 & 2 & 1,6 & 0 & 0 & 0 & 0 & 2 & 1,6 & 16 & 12,4 \\
\hline Raspador & 2 & 1,6 & 1 & 0,8 & 0 & 0 & 0 & 0 & 0 & 0 & 0 & 0 & 3 & 2,3 \\
\hline Punta & 0 & 0 & 0 & 0 & 0 & 0 & 0 & 0 & 1 & 0,8 & 0 & 0 & 1 & 0,8 \\
\hline TOTAL & 42 & 32,7 & 58 & 44,9 & 16 & 12,5 & 1 & 0,8 & 1 & 0,8 & 11 & 8,6 & 129 & 100 \\
\hline
\end{tabular}

tos confeccionados en idénticas materias primas, evidenciando que algunas lascas o instrumentos fueron extraídos a partir de determinados núcleos presentes en el conjunto.

Otro aspecto analizado en el material lítico fue la presencia de corteza. Un 70\% de los núcleos conservan corteza, como así también más del 50\% de las lascas y desechos indeterminados. Para los instrumentos, se registró presencia de corteza en más del 50\% de raederas, siendo éstos los tipos artefactuales más frecuentes, confeccionados sobre lascas. En la preforma y la punta -únicos instrumentos bifaciales del conjunto- $y$ en los tres raspadores recuperados, la corteza está totalmente ausente.

Con el fin de evaluar el tamaño más representado como así también la presencia de materiales pequeños, especialmente microlascas, se tomó en consideración la medida en milímetros del largo mayor reconocible para lascas, desechos indeterminados, raederas, raspadores y preforma (estos tres últimos formatizados siempre sobre lascas). Como puede observarse en la Figura 2, no se registraron artefactos menores a $20 \mathrm{~mm}$; este hecho es atribuible a la acción del viento, que incidiría en el grado de integridad del conjunto. Esta falta de materiales de tamaño pequeño ha sido observado con anterioridad en contextos de similares características (Borrazzo 2004, Oría et al. 2010) y también mediante experimentación controlada (Massone et al. 1993). En Amalia 4 la proporción de instrumentos es alta, contrariamente a lo que ocurre en otros contextos a nivel regional, donde prácticamente sólo se han recuperado núcleos, lascas y desechos y fue posible realizar remontajes (Santiago 2009 y bibliografía allí citada); así, este sitio, debido a su composición, podría identificarse como un locus empobrecido por la acción eólica, en el cual destaca la baja proporción de los ítems líticos de menor tamaño.

Entre los restos faunísticos ( $\mathrm{N}=35)$, la mayor parte es asignable a elementos de guanaco. El estado de preservación no permite en muchos casos llegar a una asignación sistemática a nivel de especie por falta de rasgos diagnósticos, indicando sólo Camelidae y Mammalia indet., que en general

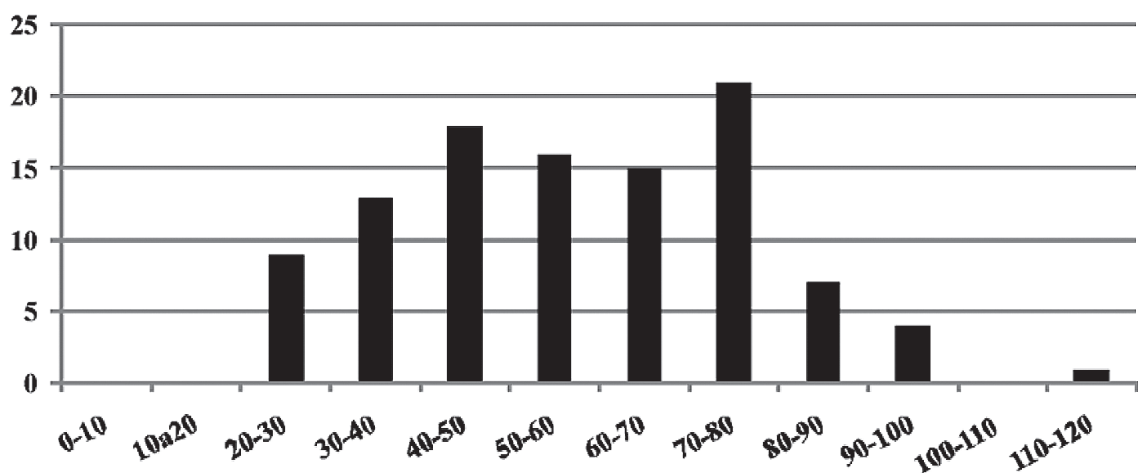

Fig. 2. Distribución de frecuencias de tamaños medida en mm, para lascas, desechos indiferenciados, raederas, raspadores y preforma. 
Tabla 2. Composición faunística en Amalia 4.

\begin{tabular}{|c|c|c|}
\hline Taxon & NISP & \%NISP \\
\hline Lama guanicoe & 14 & 40 \\
\hline Camelidae & 11 & 31,43 \\
\hline Ovis sp. & 2 & 5,71 \\
\hline Canidae & 2 & 5,71 \\
\hline Mammalia indet. & 5 & 14,29 \\
\hline Chloephaga sp. & 1 & 2,86 \\
\hline & 35 & 100 \\
\hline
\end{tabular}

son fragmentos de huesos largos con grados 4 y 5 de meteorización (sensu Behrensmeyer 1978). De todas maneras, considerando la componente faunística de la región, es altamente probable que todo ese material sea asignable a guanaco. En la tabla 2 se detalla la composición taxonómica del conjunto; allí puede observarse que -sumados- Camelidae y Lama guanicoe superan el $80 \%$ de la muestra, representando un $\mathrm{MNI}=2$. Los restos recuperados corresponden al esqueleto apendicular, particularmente huesos largos, en tanto el esqueleto craneano está representado por dos molares. A excepción de unos pocos restos -entre los cuales se encuentran los de Canidae y de Chloephaga sp. y un fragmento de tibia asignable a Camelidae-, que presentan una superficie totalmente blanquecina, el resto del material ha perdido el tejido cortical y cualquier tipo de marca queda así borrada. Los especímenes con evidencias culturales son muy escasos.

Un fragmento de diáfisis de tibia de guanaco podría interpretarse como un retocador, pero la superficie está demasiado alterada como para identificar pulidos u otra clase de procesamiento sobre el mismo. Queda por el momento planteado como hipótesis a contrastar.
Teniendo en cuenta la ubicación de la cubeta de deflación en el paisaje, el afloramiento de un estrato rico en guijarros que pudieron haber sido utilizados como materia prima para la talla -tal como se ve también en las costas altas de otras lagunas del área de estudio- y la diferencia numérica entre restos óseos y líticos, se planteó en primera instancia que se trataría de un contexto de tipo taller. En relación al uso del espacio, para la región se ha esbozado un patrón en trabajos previos (Oría 2009, Oría et al. 2010, Coronato et al. 2009), notándose en este tipo de costas lagunares poca evidencia de otras actividades. En Amalia 4 la alta frecuencia de instrumentos, la baja proporción de especímenes de pequeño tamaño y el alto grado de meteorización del material óseo dan como resultado una baja resolución del conjunto y se requiere de un análisis tafonómico más fino -tanto del conjunto lítico (sensu Borrazzo 2004), como en el conjunto faunístico- para contemplar también la integridad del mismo.

\section{La punta de obsidiana verde}

Este instrumento merece una consideración particular pues resulta un indicador clave no sólo por su morfología sino fundamentalmente por la materia prima en la que fue confeccionada y las implicancias en su distribución regional. La punta recuperada en Amalia 4 fue confeccionada sobre obsidiana verde oscura; es de tipo pedunculada de limbo triangular largo, con ápice normal y aletas destacadas ligeramente asimétricas (Fig. 3). El pedúnculo es claramente diferenciado de lados convergentes cóncavos y de base recta, siguiendo la clasificación propuesta por Orquera y Piana (1986).

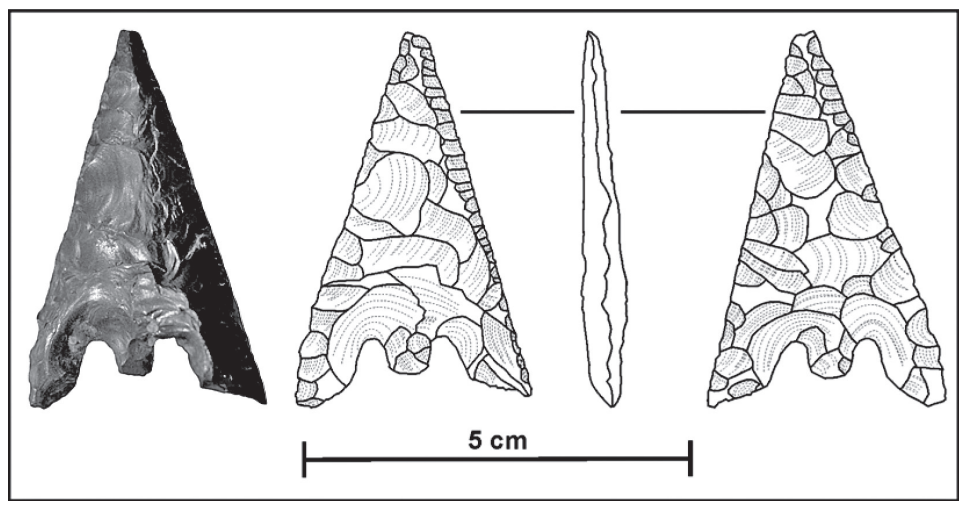

Fig. 3. Punta de obsidiana verde de sitio Amalia 4. 
Su estado de conservación es bueno, faltándole un pequeño fragmento en el ápice. No se encuentra erosionada, ya que las aristas en los negativos de retoque están bien definidas; tampoco se observan pátinas en su superficie. Sus dimensiones son: 41 $\mathrm{mm}$ de longitud de la punta a la base del pedúnculo y $45 \mathrm{~mm}$ tomando el largo total de la pieza (esto es, incluyendo las aletas); ancho máximo $23 \mathrm{~mm}$ y 5 mm de espesor, con un peso de 3,8 g.

La morfología de la punta podría corresponderse con las de igual materia prima recuperadas por Morello y colaboradores (2001) en el sitio Río Verde (280 \pm 60 años AP), difiriendo notablemente de las puntas de obsidiana verde de los sitios tempranos, como Englefield, en el sector de mares interiores, donde las puntas pedunculadas son una rareza (Emperaire y Laming 1961). Considerando entonces la cronología dada en Río Verde y las características morfológicas de esta punta, se podría referir el marco temporal para el contexto de Amalia 4, o al menos parte del mismo, a fines del Holoceno, siendo éste similar a otros ya analizados en la región.

\section{DISCUSIÓN}

El registro de obsidiana verde en sitios de cazadores recolectores del extremo sur de Patagonia, tanto chilena como argentina, ha sido objeto de numerosos estudios (entre otros Johnson 1976, Stern y Prieto 1991, Stern 2000, Morello et al. 2001, Manzi 2004). Si bien la fuente de esta materia prima continúa sin ser ubicada, reiterados análisis de la misma revelan que se trata de una fuente única y su procedencia pudo ser acotada al sector de mares interiores (Senos Otway y Skyring) próximo al estrecho de Magallanes (Stern y Prieto 1991, Stern 2000, Morello et al. 2001).

Si bien son varios los registros de esta materia prima en contextos de cazadores terrestres, pocos se conocen en la estepa norte de la Isla Grande de Tierra del Fuego. En las costas de la bahía Inútil (Fig. 1b), o cercanos a ella, se registró obsidiana verde en la localidad Marazzi: dos lascas en el sitio Marazzi 1 (Morello et al. 1999, Morello et al. 2010), una lasca en el sitio Marazzi 13, Bloque de la Obsidiana (Morello et al. 2009), otra en el sitio Marazzi 2 (Calas y Lucero 2009) y una lasca con retoque en Marazzi 22 (Morello et al. 2011). Otros registros de obsidiana verde en las costas de Tierra del Fuego provienen del sitio Porvenir Norte 28, con una punta de proyectil y una lasca en el sitio Río Caleta 4 (Morello et al. 2011). De particular interés son los casos en que se registra obsidiana verde en sitios del interior de la estepa fueguina, lejos de las redes de circulación canoera. Este es el caso de una lasca de obsidiana verde recuperada cerca de la laguna Grande y otra descripta para el conjunto lítico del sitio Avilés 3 (Fig. 1b; Santiago 2009) y un pequeño núcleo fragmentado en el sitio Puesto Consuelo 3 (Morello et al. 2011). A estos registros sumamos ahora la punta de Amalia 4.

La importancia del estudio de la obsidiana verde como materia prima radica en su característica de bien de intercambio. Es una evidencia clara de algún tipo de contacto entre las poblaciones canoeras y las poblaciones de cazadores terrestres ya que su fuente debió estar disponible únicamente para los grupos que empleaban canoas para desplazarse. En este sentido consideramos a esta materia prima como "exótica" para los grupos pedestres. Este planteo se ve fortalecido además por la diferencia entre los tipos artefactuales recuperados en los sitios. Morello y colaboradores (2004) observan en sitios ubicados en el área de los mares interiores las mayores cifras en frecuencias de hallazgos, peso y variedad de artefactos, describiendo una estrategia expeditiva en el uso de la obsidiana verde (Morello et al. 2004). Para contextos de cazadores terrestres, por el contrario, los mismos autores plantean una estrategia de curaduría o conservación en base a la cantidad, tamaño y peso de las piezas (Morello et al. 2004).

Dada la amplitud del área en que se registra la presencia de obsidiana verde, queda manifestada la magnitud de las redes de intercambio establecidas -aun con interrupciones- a nivel macro regional. Uno de los principales motivos que incentivarían este tipo de contactos es la disminución del riesgo a través de sistemas de alianzas en momentos de escasez (Neme y Gil 2005). En este sentido los ítems exóticos, cobran importancia al representar aquellos contactos que se establecen con el propósito de mantener dichas alianzas regionales. Este aspecto es lo que le confiere visibilidad arqueológica a las redes, a través de la circulación de elementos que tienen significado simbólico (Whallon 2006); por ejemplo, el hallazgo de piezas de obsidiana verde en contexto de enterratorios humanos sobre el es- 
trecho de Magallanes evidencia el carácter simbólico o al menos la importancia que se le confiere a esta materia prima fuera del área de su fuente.

\section{CONSIDERACIONES FINALES}

El registro de obsidiana verde para el norte de la isla Grande de Tierra del Fuego, se da únicamente en contextos tardíos. La ausencia de obsidiana verde en este sector para el bloque temporal del Holoceno medio -6.000 a 4.000 años AP-, siendo que está presente en lugares tan distantes como el campo volcánico de Pali Aike y el canal Beagle, podría deberse a una baja demografía para eso lapso, sin descontar un posible sesgo de muestreo para fechas tempranas, dado el escaso desarrollo de la investigación arqueológica en determinados sectores de la isla. En la estepa fueguina, la cantidad de sitios con fechados tardíos es muy superior a los tempranos. Siguiendo a Gamble (1993) el número de alianzas variará con el tiempo por cambios en las condiciones ecológicas o por fluctuaciones de las poblaciones locales. Si bien no hay aún suficiente evidencia para sostener un incremento poblacional, para momentos tardíos la ocurrencia de obsidiana verde en sectores inaccesibles para grupos canoeros en la isla de Tierra del Fuego, como es el caso de la punta recuperada en el sitio Amalia 4, sería un indicador de mayor interacción entre grupos.

\section{AGRADECIMIENTOS}

Matías Bitch y Martín Occhionero de la Ea. San Julio facilitaron el acceso al lugar de trabajo, brindando apoyo durante la estadía en el campo; Rocío Blanco (UNLP) y Alejandra Raies (UNR), colaboraron en el trabajo de campo, Nélida Pal orientó en el análisis tecno-morfológico del conjunto lítico y Martín Vázquez y tres revisores anónimos contribuyeron a mejorar este manuscrito. Este trabajo ha sido financiado por el PICT 200538096 (ANPCyT). Los autores son únicos responsables de lo aquí expresado.

\section{BIBLIOGRAFÍA}

BEHRENSMEYER, A.K. 1978. Taphonomic and ecologic information from bone weathering. Paleobiology (4): 150-162.
BORRAZZO, K. 2004. Hacia una tafonomía lítica: el análisis tafonómico y tecnológico de los conjuntos artefactuales líticos de superficie provenientes de los loci San Genaro 3 y 4 (Bahía San Sebastián - Tierra del Fuego, Argentina). Tesis de licenciatura en Ciencias Antropológicas, Facultad de Filosofía y Letras, Universidad de Buenos Aires. Ms.

CALAS, E. y M. LUCERO. 2009. El sitio Marazzi 2 (Tierra del Fuego): una ocupación costera de cazadores terrestres. En: Arqueología de la Patagonia. Una mirada desde el último confín. Salemme M., F. Santiago, M. Álvarez, E. Piana, M. Vázquez y E. Mansur (eds.), Tomo II: 957-976. Editorial Utopías. Ushuaia.

CORONATO A., FANNING P., SALEMME M., ORÍA J., y J. PICKARD. 2009. Aeolian paleosols and the archaeological record at Lake Arturo, Northern Tierra del Fuego, Argentina. IV Congreso de Geomorfología y Geología del Cuaternario. Resumen 234. La Plata.

EMPERAIRE, J. y A. LAMING. 1961. Les gisements des iles Englefield et Vivian dans la mer d'Otwey (Patagonie austral). Journal de la Société des Américanistes 50:777. París. Traducción L. Orquera.

FANNING, P. y S. HOLDAWAY. 2001. Temporal limits to the archaeological record in arid western NSW, Australia: lessons from OSL and radiocarbon dating of hearths and sediments. En Australasian connections and new directions: proceedings of the 7th Australasian Archaeometry Conference. Research in Anthropology \& Linguistics 5, Jones M. y Sheppard P. (eds): 85-104, University of Auckland.

GAMBLE, C. 1993. Exchange, foraging and local hominid networks. En Trade and exchange in prehistoric Europe, Scarre C. y F. Healy (eds): 35-45, Oxbow Bocks.

HOLDAWAY, S. J., J. SHINER Y P. FANNING. 2004. Huntergatherers and the archaeology of discard behavior: an analysis of surface stone artifacts from Sturt National Park, western New South Wales, Australia. Asian Perspectives. 43 (1):34-72.

JOHNSON L. 1976. Informe sobre una prospección arqueológica en Magallanes. Anales del Instituto de la Patagonia 7:87-94. Punta Arenas.

MANZI L. 2004. La obsidiana verde como indicador de formas de uso del espacio en Fuego-Patagonia. En Contra viento y marea Arqueología de Patagonia. Editado por Civalero M. T., P. Fernandez y G. Guraieb, 149-166. INAPL. Sociedad Argentina de Antropología. Buenos Aires.

MASSONE, M., D. JACKSON y A. PRIETO. 1993. Perspectiva arqueológica de los Selk'nam. Centro de Investigaciones Diego Barros Arana, Dirección de Bibliotecas, Archivos y Museos. Santiago de Chile. 
MORELLO, F., CONTRERAS, L. y SAN ROMÁN, M. 1999. La localidad Marazzi y el sitio arqueológico Marazzi I, una reevaluación. Anales del Instituto de la Patagonia, Serie Ciencias Humanas 27: 183-197. Punta Arenas. MORELLO, F., M. SAN ROMÁN, A. PRIETO y C. STERN. 2001. Nuevos antecedentes para una discusión arqueológica en torno a la obsidiana verde en Patagonia Meridional. Anales del Instituto de la Patagonia, Serie Ciencias Humanas, 29: 129-148. Punta Arenas.

MORELLO, F., M. SAN ROMÁN y A. PRIETO. 2004. Obsidiana verde en Fuego-Patagonia: distribución y estrategias tecnológicas. Contra viento y marea. Arqueología de Patagonia. Editado por Civalero M. T., P. Fernandez y G. Guraieb, 149-166. INAPL. Sociedad Argentina de Antropología. Buenos Aires.

MORELLO, F., L. A. BORRERO, J. TORRES, M. MASSONE, M. ARROYO, R. MCCULLOCH, E. CALÁS, M. J. LUCERO, I. MARTÍNEZ y G. BAHAMONDE. 2009. Evaluando el registro arqueológico de Tierra del Fuego durante el Holoceno temprano y medio. Arqueología de la Patagonia. Una mirada desde el último confín. Editado por Salemme M., F. Santiago, M. Álvarez, E. Piana, M. Vázquez y E. Mansur, II. 1031-1047. Editorial Utopías. Ushuaia.

MORELLO F., BORRERO L., MASSONE M., STERN CH., GARCÍA-HERBST A., McCULLOCH R., ARROYOKALIN M., CALÁS E., TORRES J., PRIETO A., MARTÍNEZ I., BAHAMONDE G. y P. CÁRDENAS. 2011. The Holocene peopling of Tierra del Fuego: discussing colonization, biogeographic barriers and interactions in southernmost Patagonia, Chile. Antiquity. En prensa

NEME G. y A. GIL. 2005. Aportes para la discusión del intercambio en el sur de Mendoza. En Actas del XVI Congreso Nacional de Arqueología Chilena. Chile.
ORÍA, J. 2009. Prospecciones en el interior de la estepa fueguina. Arqueología de la Patagonia. Una mirada desde el último confín. Editado por Salemme M., F. Santiago, M. Alvarez, E. L. Piana, M. Vázquez y M. E. Mansur, II. 1065-1082. Editorial Utopías. Ushuaia.

ORÍA, J., M. SALEMME, F. SANTIAGO y A. MONTES. 2010. Localidad arqueológica Tres Marías: un caso para evaluar la pérdida de material arqueológico por erosión eólica en la estepa fueguina. Cazadores-Recolectores del Cono Sur. Revista de Arqueología, Vol. 4. Mar del Plata. En prensa.

ORQUERA, L. y E. PIANA. 1986. Normas para la descripción de objetos arqueológicos de piedra tallada. CADIC, Contribución Científica (Publicación especial) $\mathrm{N}^{\circ} 1$, Ushuaia. Pp: 198

SANTIAGO, F. 2009. La ocupación humana del norte de Tierra del Fuego durante el Holoceno medio y tardío. Su vinculación con el paisaje. Tesis de Doctorado en Arqueología, Universidad Nacional del Centro, Olavarría. Ms.

SANTIAGO, F. y J. ORÍA. 2007. Lo que el viento no se llevó. Análisis de sitios de superficie en la estepa fueguina. Magallania. 35 (2):117-128. Punta Arenas.

STERN, CH. 2000. Fuentes de los artefactos de obsidiana en los sitios arqueológicos de las cuevas de Pali Aike y Fell, y Cañadón La Leona, en Patagonia Austral. Anales del Instituto de la Patagonia, Serie Ciencias Humanas, 28:251-263. Punta Arenas.

STERN, CH. y A. PRIETO. 1991. Obsidiana verde de los sitios arqueológicos en los alrededores del seno Otway, Magallanes, Chile. Anales del Instituto de la Patagonia, Serie Ciencias Sociales, 20:139-144. Punta Arenas.

WHALLON, R. 2006. Social networks and information: non"utilitarian" mobility among hunter-gatherers. Journal of Anthropological Archaeology 25:259-270. 
